\title{
Vitamin B1 and B6 in the malaria parasite: requisite or dispensable?
}

\author{
C. Wrenger, J. Knöckel, R.D. Walter and I.B. Müller \\ Department of Biochemistry, Bernhard Nocht Institute for Tropical Medicine, Hamburg, Germany \\ Correspondence to: C. Wrenger, Department of Biochemistry, Bernhard Nocht Institute for Tropical \\ Medicine, Bernhard Nocht Strasse 74, D-20359 Hamburg, Germany \\ Fax: +49-40-4281-8418. E-mail: wrenger@bni-hamburg.de
}

\begin{abstract}
Vitamins are essential compounds mainly involved in acting as enzyme co-factors or in response to oxidative stress. In the last two years it became apparent that apicomplexan parasites are able to generate B vitamers such as vitamin B1 and B6 de novo. The biosynthesis pathways responsible for vitamin generation are considered as drug targets, since both provide a high degree of selectivity due to their absence in the human host. This report updates the current knowledge about vitamin B1 and B6 biosynthesis in malaria and other apicomplexan parasites. Owing to the urgent need for novel antimalarials, the significance of the biosynthesis and salvage of these vitamins is critically discussed in terms of parasite survival and their exploitation for drug development.
\end{abstract}

Key words: Malaria; Apicomplexa; Pyridoxal phosphate synthase; Vitamin B6; Vitamin B1

Presented at the XXXVI Annual Meeting of the Sociedade Brasileira de Bioquímica e Biologia Molecular, Salvador, BA, Brazil, May 21-25, 2007.

Research supported by Deutsche Forschungsgemeinschaft Grant WA 395/15.

Received September 18, 2007. Accepted December 6, 2007

\section{Introduction}

Apicomplexan parasites have a significant impact on human and livestock health, and chemotherapy remains a problem. The most important apicomplexan parasite is Plasmodium, the pathogenic agent of malaria. Malaria is a devastating and quite often a deadly parasitic disease which causes important public health problems in the tropics. However, coccidiosis caused by Cryptosporidia and Toxoplasma infections also pose a serious threat especially to immunocompromised people such as HIV patients all over the world and first infection with Toxoplasma during pregnancy can lead to developmental damage to the fetus.

Due to the high mutational rate of Plasmodium falciparum and its resulting rapid adaptation to environmental changes, drug resistance to the standard medication with chloroquine and antifolates is increasing. Therefore, continuous discovery of novel drug targets and development of new chemotherapeutic agents are inevitable. It has been claimed that antiparasitic compounds should preferably be created to target only the parasite without harming the human host. In this respect, the biosynthesis of parasite-specific vitamin B1 and B6 represents an ideal drug target.

\section{Alternative pathways of vitamin B6 biosynthesis}

Two different pathways for the synthesis of vitamin B6 are currently known. While 4-phosphohydroxy-L-threonine and 1-deoxyxylulose 5'-phosphate (DOXP) are substrates in the Escherichia coli (DOXP-dependent) pathway $(1,2)$, vitamin B6 is synthesized from ribose 5'-phosphate, glyceraldehyde 3'-phosphate and glutamine in a DOXP-independent or fungus-like pathway (3-8). The DOXP-independent pathway has been identified in fungi, plants and some bacteria and was originally considered to be in- 
volved in the detoxification of singlet oxygen $\left({ }^{1} \mathrm{O}_{2}\right)(3,9)$. However, the analysis of fungal mutants deficient in singlet oxygen resistance (SOR1), and therefore sensitive to singlet oxygen, demonstrated that the product of this gene also participates in pyridoxine biosynthesis $(3,4,9)$. The SOR1 enzyme (also named $\mathrm{Pdx} 1$ ) belongs to the highly conserved enzyme family SNZ in Saccharomyces cerevisiae. The SNZ1 protein has been shown to interact with the SNO1 protein (5), also named Pdx2, which is a member of another preserved family in yeast consisting of three SNO enzymes (10). The involvement of $\mathrm{Pdx} 2$ in de novo vitamin B6 synthesis was confirmed by complementation assays of mutants deficient in pyridoxine biosynthesis (11). This pathway for vitamin B6 biosynthesis has been demonstrated in various other organisms such as Bacillus subtilis and Arabidopsis thaliana $(6,9,12-14)$. In contrast to the $E$. coli-like formation of vitamin B6 leading to pyridoxine 5'phosphate, the synthesis of the latter pathway results directly in pyridoxal phosphate (PLP), the active form of vitamin B6.

A few years ago Cassera et al. (15), using labeling experiments, suggested the presence of a vitamin B6 metabolite in $P$. falciparum which we later reported to be produced via the DOXP-independent pathway (16) (Figure 1). Moreover, we demonstrated a homologous pathway in the other apicomplexan parasite Toxoplasma gondii, where PLP synthesis also is a product of the single copy genes $P d x 1$ and Pdx2 (17). The two proteins need to act together for enzyme activity, similar to the homologous yeast proteins SNZ1 and SNO1 (6). The functionality of the two proteins of the apicomplexan parasites was verified by complementation of vitamin B6-deficient yeast strains (16). As is the case for yeast, in apicomplexa vitamin B6 is directly synthesized in its active form $\operatorname{PLP}(7,8,17,18)$. The $\mathrm{Pdx} 1$ protein is responsible for the creation of PLP by utilizing pentose, triose as well as ammonia. The ammonia is provided by the $\mathrm{Pdx} 2$ protein, which possesses glutaminase activity. Interestingly, the activity of $\mathrm{Pdx} 1$ is not dependent on the presence of Pdx2 if glutamine is replaced by ammonia, whereas the activity of $\mathrm{Pdx} 2$ relies on its interaction with $\mathrm{Pdx} 1(16,17)$.

The crystal structures of the $B$. subtilis and Geobacillus stearothermophilus $\mathrm{Pdx} 1$ and $\mathrm{Pdx} 2$ homologues have already been solved $(19,20)$. The structures of the entire $\mathrm{Pdx} 1 / \mathrm{Pdx} 2$ complex from $B$. subtilis and Thermotoga maritima have been analyzed only recently, with the identification of PLP synthase as a multimeric protein complex consisting of $12 \mathrm{Pdx} 1$ proteins decorated by $12 \mathrm{Pdx} 2$ proteins $(21,22)$. These data suggest a channel between the individual $\mathrm{Pdx} 1$ and $\mathrm{Pdx} 2$ proteins for the transfer of the ammonia produced from Pdx2 towards the active site of $P d x 1$. Amino acid residues involved in the enzyme activity of $\mathrm{Pdx} 1$ proteins $(21,23)$ are also present in the $\mathrm{Pdx} 1$ enzymes of the apicomplexan parasites $P$. falciparum and T. gondii. Interestingly, analyses of the genome data available for Eimeria or Cryptosporidia and for other protozoan parasites such as Trypanosoma and Leishmania did not reveal any indices for genes involved in de novo vitamin B6 synthesis. This clearly indicates that PLP biosynthesis is not a general event in protozoan parasites. Parasites which are dependent on external sources of vitamin B6 need to take up this nutrient like the human host. After import, B6 vitamers are immediately phosphorylated and thereby trapped within the cell. This catalysis is carried out by pyridoxine kinase (PdxK) (24-27). In addition to performing de novo synthesis, the malaria parasite also holds

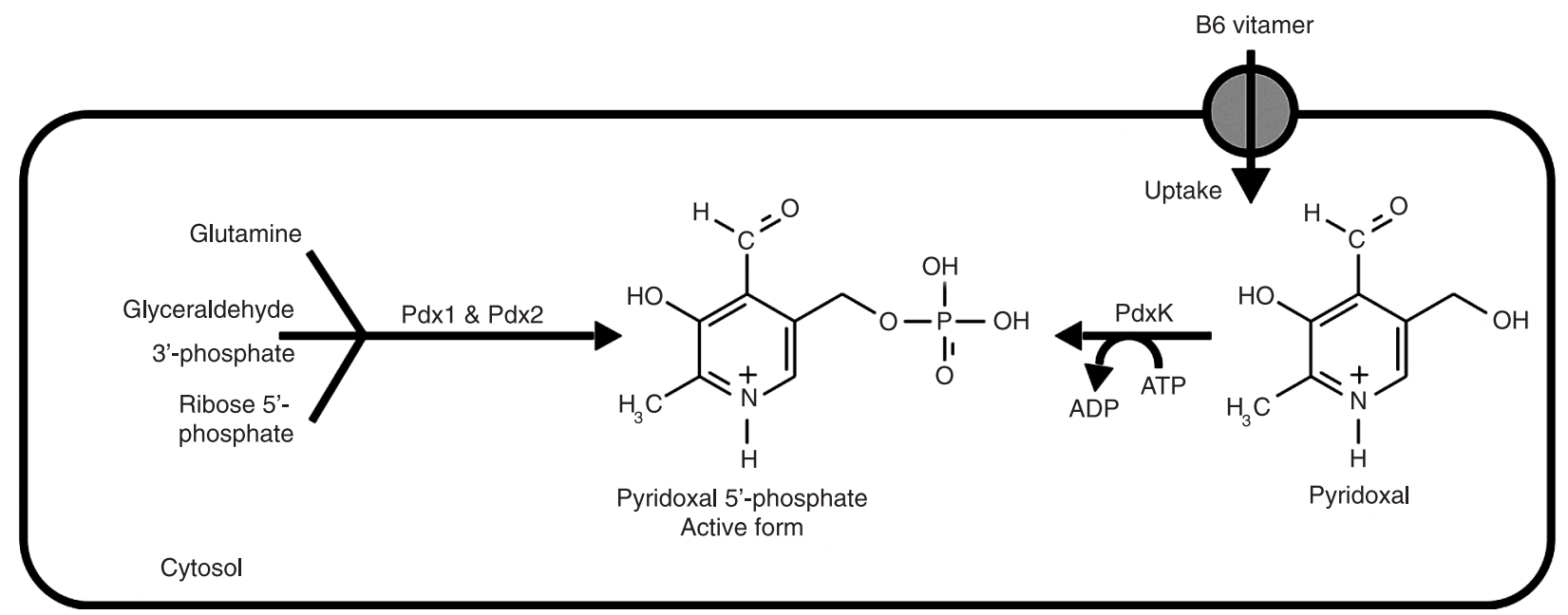

Figure 1. Vitamin B6 biosynthesis in Plasmodium falciparum. For explanation of the abbreviations see text. 
a PdxK which enables $P$. falciparum to salvage $B 6$ vitamers (16) (Figure 1). However, the value of the dual PLP provision in Plasmodium is not known and needs to be further analyzed.

\section{Suitability of vitamin B6 biosynthesis for chemotherapy}

The question arises whether vitamin B6 metabolism can be exploited to develop novel antimalarials. In general, there are two options to interfere with the biosynthesis of plasmodial vitamin B6. i) Since the crystal structure of PLP synthase was solved for $T$. maritima and $B$. subtilis, the design of inhibitors targeting this enzyme complex in order to block biosynthesis was facilitated $(21,22)$. Nevertheless, rational drug design will benefit from structural analyses of the plasmodial PLP synthase crystal to detect parasite-specific targets. ii) On the other hand, emphasis should also be placed on unphosphorylated B6 vitamers, which after uptake are phosphorylated by PdxK and trapped within the parasite (16). Derivatives of these vitamers offer the strategy of channeling inactive co-factors into the depot of PLP, thereby poisoning PLP-dependent enzymes. Both strategies have a high potential for the design of novel drugs in order to interfere with the parasite's vitamin B6 metabolism.

\section{Vitamin B1 and its biosynthesis}

Until recently only prokaryotes, fungi and plants were reported to synthesize vitamin B1. Mammals entirely depend on the salvage of vitamin B1 from their diet. The active form of vitamin B1 is thiamine pyrophosphate (TPP) which is a co-factor for various enzymes mainly involved in carbohydrate metabolism such as 2-oxoglutarate dehydrogenase, pyruvate dehydrogenase or transketolase $(28,29)$. The biosynthesis of thiamine occurs by the combination of two different branches. The pyrimidine branch provides 4-amino-5-hydroxymethyl-2-methylpyrimidine (HMP), which has to be phosphorylated to HMP diphosphate (HMP-PP) in two consecutive steps by HMP/HMP-P kinase (ThiD; Figure 2) (30-33). In bacteria HMP itself is formed from precursors of the purine biosynthesis by the HMP synthesis enzyme ThiC (34), whereas in yeast the Thi5 protein is suggested to link pyridoxine 5'-phosphate and histidine to form HMP-P (35-37). The product of the other branch is 5-(2-hydroxyethyl)-4-methylthiazole phosphate (THZ-P), which can also be provided by the salvage of THZ upon phosphorylation by THZ kinase (ThiM) $(38,39)$. Subsequently, the respective phosphorylated pyrimidine and thiazole moieties, HMP-PP and THZ-P, are amalgam- ated by thiamine phosphate synthase (ThiE) to form thiamine phosphate (TMP) (40). An additional phosphorylation step by TMP kinase (ThiL) would be necessary for this to become the active diphosphorylated form (32). However, in yeast as well as in some bacteria ThiL is absent. Therefore, it has been suggested that TMP is first dephosphorylated by a phosphatase before it is pyrophosphorylated by thiamine pyrophosphokinase (TPK) $(41,42)$. The malaria parasite contains the genes encoding for the vitamin B1 synthesis enzymes ThiM, ThiD, and ThiE and their expression and functionality was confirmed $(33,43)$ (Figure 2). Consistent with the situation in other organisms, an open-reading frame encoding for ThiL is not found in the plasmodial genome database.

\section{Creation of a "thiamine pool" in plasmodium falciparum}

Instead of ThiL, a gene with homology to TPK has been identified $(43,44)$ and analyzed for its kinetic parameters (45) (Figure 2). The plasmodial TPK does not accept the de novo synthesized TMP as substrate, which means that TMP must first be dephosphorylated to thiamine before it is pyrophosphorylated to TPP by PfTPK (45). The dephosphorylation of TMP is thought to be carried out by a nonspecific phosphatase (46). Recently, such a phosphatase was characterized and its substrate specificity for PLP and TMP shown (Knöckel J, Müller IB, Walter RD, Wrenger C, unpublished data).

Pyrophosphorylation of thiamine to TPP takes place solely in the cytosol of the parasite, as demonstrated by immunofluorescence assays of PfTPK (45). However, TPPdependent enzymes are located not only in the cytosol but also in different compartments, a fact that raises the question of TPP transport in these plasmodial organelles (Figure 2). For instance, the TPP-dependent pyruvate dehydrogenase complex is targeted to the apicoplast, whereas the 2-oxoglutarate dehydrogenase complex has been reported to be mitochondrial $(47,48)$. Assuming that thiamine is pyrophosphorylated exclusively in the cytosol, $P$. falciparum must hold specific transporters to fulfill the need for TPP of both organelle-specific dehydrogenase complexes. The relocation of TPP into the yeast mitochondrion by $a$ specific transporter has been reported (49).

\section{Does plasmodial vitamin B1 biosynthesis serve as a target for chemoterapy?}

Promising attempts to exploit vitamin B1 biosynthesis for chemotherapy have been made in bacteria by using the naturally occurring HMP analogue bacimethrin (MeO-HMP) 
or the synthetic $\mathrm{CF}_{3}-\mathrm{HMP}$ compound $(50,51)$. Although the former compound was successfully tested as a substrate for the plasmodial ThiD protein, bacimethrin did not affect the proliferation rate of the parasite, a fact that could be explained by an inefficient salvage of this analogue (33). Nevertheless, HMP derivatives as such should be considered as promising drug candidates against the malaria parasite, as already shown for bacteria $(33,51)$.

Culturing $P$. falciparum in thiamine-free medium for a few days showed no adverse effect on parasite growth behavior (52); however, extending the period of culture in vitamin B1-deficient medium to ten days revealed a significant need for the externally supplied thiamine precursor HMP or thiamine itself for growth (33). The necessity of HMP in the medium clearly indicates that the parasite is able to create thiamine but depends on added HMP. Consistent with these results a homologue of the yeast-like Thi5 enzyme was not found in the plasmodial genome database, indicating the absence of a linkage of the vitamin $B 6$ and $B 1$ biosynthesis pathways $(36,53-55)$. Furthermore, there is no plasmodial homologue coding for the bacterial ThiC protein, the enzyme that catalyzes the formation of HMP from aminoimidazole ribonucleotide, an intermediate of purine synthesis (32). The latter is not surprising since purine biosynthesis is absent in the malaria parasite (56). Considering that Plasmodium can either salvage HMP - as mentioned above - or thiamine for the formation of TPP offers novel chemotherapeutic strategies to import pro-drugs in the form of HMP or thiamine derivatives. Indeed, pyrithiamine and thiochrome, both

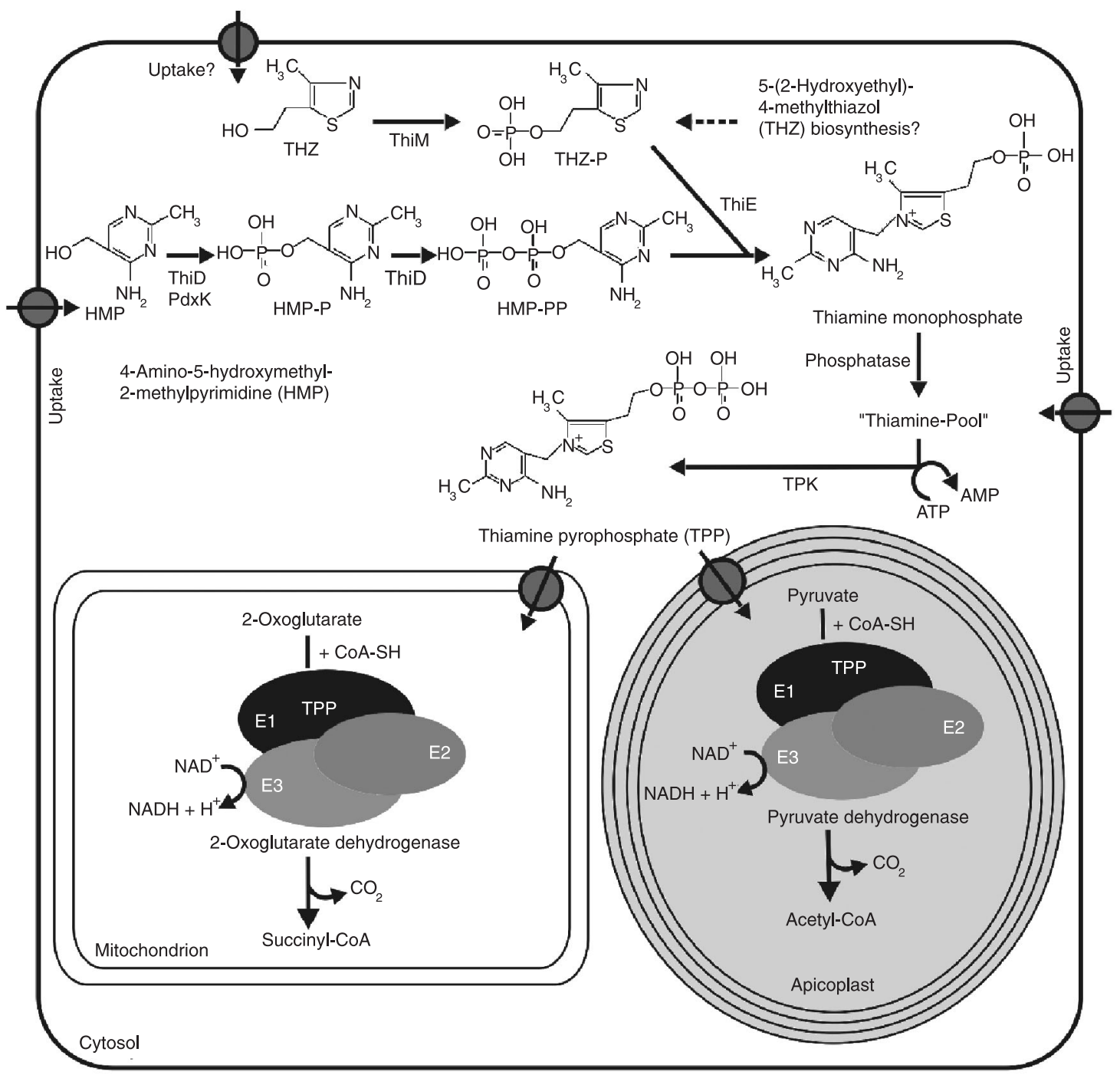

Figure 2. Generation of thiamine pyrophosphate and its trafficking within Plasmodium falciparum. Transport mechanisms are indicated by $\AA$. For explanation of the abbreviations see text. 
thiamine derivatives, were tested as substrates for plasmodial TPK. While pyrithiamine is accepted equivalently to thiamine as substrate, thiochrome is not phosphorylated by PfTPK (45). However, testing pyrithiamine on cultured parasites did not reveal any anti-proliferative effect even at a concentration of $0.5 \mathrm{mM}$, implying an insufficient uptake of this analogue by the parasite or poor targeting of vitamin B1-dependent enzymes by its pyrophosphorylated form (Müller IB, Walter RD, Wrenger C, unpublished data).

Interestingly, cultivation of the parasite in THZ-deficient medium led to normal growth, suggesting that $P$. falciparum might be able to generate $\mathrm{THZ}$ de novo. Despite the use of bioinformatic tools, genes coding for $\mathrm{THZ}$ biosynthesis enzymes could not be identified in the plasmodial genome database (57). The synthesis of either the pyrimidine or the thiazole moiety was discussed for various other organisms, requiring the other part to be salvaged $(28,58,59)$. This might be also the case for the malaria parasite; however, we cannot exclude a THZ biosynthesis pathway different from the prokaryotic pathway in $P$. falciparum.

\section{Does vitamin B1 biosynthesis occur in other apicomplexan parasites?}

Enzymes involved in vitamin B1 synthesis as shown for
$P$. falciparum are apparently absent in the genome of the apicomplexan relatives E. tenella (http://www.sanger.ac.uk/ Projects/E_tenella/), and T. gondii (http://www.toxodb.org/ toxo/home.jsp) (17). This major difference poses questions about evolutionary adaptation of these parasites to their hosts. Assuming that the ancestor of apicomplexa had the potential to synthesize vitamin B1 de novo or at least to salvage the THZ- and HMP-moieties, apicomplexan parasites like $T$. gondii have lost their ability to synthesize vitamin B1 de novo during evolution. Therefore, these parasites entirely depend on thiamine uptake to satisfy their needs, a fact that might be explained by their perfect accommodation to their host cells. The malaria parasite, however, still expresses a rudimentary set of vitamin B1 synthesis enzymes pointing out an insufficient vitamin B1 supply from its host. This distinct adaptation of apicomplexan parasites may result from life cycle and host cell specificity. Further analyses are required to solve the riddle concerning de novo co-factor synthesis, uptake and salvage.

In summary, vitamins B1 and B6 are essential cofactors and their biosyntheses offer a powerful potential as drug targets, since these pathways are absent in the human host. Ongoing studies on the structures of the enzymes involved in vitamin B1 and B6 synthesis will allow the design of specific inhibitors blocking these crucial pathways and thereby impairing parasite growth.

\section{References}

1. Roa BB, Connolly DM, Winkler ME. Overlap between pdxA and ksgA in the complex pdxA-ksgA-apaG-apaH operon of Escherichia coli K-12. J Bacteriol 1989; 171: 4767-4777.

2. Lam HM, Tancula E, Dempsey WB, Winkler ME. Suppression of insertions in the complex pdxJ operon of Escherichia coli K-12 by lon and other mutations. J Bacteriol 1992; 174: 1554-1567.

3. Ehrenshaft M, Jenns AE, Chung KR, Daub ME. SOR1, a gene required for photosensitizer and singlet oxygen resistance in Cercospora fungi, is highly conserved in divergent organisms. Mol Cell 1998; 1: 603-609.

4. Osmani AH, May GS, Osmani SA. The extremely conserved pyroA gene of Aspergillus nidulans is required for pyridoxine synthesis and is required indirectly for resistance to photosensitizers. J Biol Chem 1999; 274: 23565-23569.

5. Padilla PA, Fuge EK, Crawford ME, Errett A, Werner-Washburne $\mathrm{M}$. The highly conserved, coregulated SNO and SNZ gene families in Saccharomyces cerevisiae respond to nutrient limitation. J Bacteriol 1998; 180: 5718-5726.

6. Dong YX, Sueda S, Nikawa J, Kondo H. Characterization of the products of the genes SNO1 and SNZ1 involved in pyridoxine synthesis in Saccharomyces cerevisiae. Eur J Biochem 2004; 271: 745-752.
7. Burns KE, Xiang Y, Kinsland CL, McLafferty FW, Begley TP. Reconstitution and biochemical characterization of a new pyridoxal-5'-phosphate biosynthetic pathway. J Am Chem Soc 2005; 127: 3682-3683.

8. Raschle T, Amrhein N, Fitzpatrick TB. On the two components of pyridoxal 5'-phosphate synthase from Bacillus subtilis. J Biol Chem 2005; 280: 32291-32300.

9. Ehrenshaft M, Bilski P, Li MY, Chignell CF, Daub ME. A highly conserved sequence is a novel gene involved in de novo vitamin B6 biosynthesis. Proc Natl Acad Sci U S A 1999; 96: 9374-9378.

10. Rodriguez-Navarro $S$, Llorente $B$, Rodriguez-Manzaneque MT, Ramne A, Uber G, Marchesan D, et al. Functional analysis of yeast gene families involved in metabolism of vitamins B1 and B6. Yeast 2002; 19: 1261-1276.

11. Ehrenshaft M, Daub ME. Isolation of PDX2, a second novel gene in the pyridoxine biosynthesis pathway of eukaryotes, archaebacteria, and a subset of eubacteria. J Bacteriol 2001; 183: 3383-3390.

12. Mittenhuber G. Phylogenetic analyses and comparative genomics of vitamin B6 (pyridoxine) and pyridoxal phosphate biosynthesis pathways. J Mol Microbiol Biotechnol 2001; 3: $1-20$. 
13. Belitsky BR. Physical and enzymological interaction of Bacillus subtilis proteins required for de novo pyridoxal 5'phosphate biosynthesis. J Bacteriol 2004; 186: 1191-1196.

14. Tambasco-Studart M, Titiz O, Raschle T, Forster G, Amrhein N, Fitzpatrick TB. Vitamin B6 biosynthesis in higher plants. Proc Natl Acad Sci U S A 2005; 102: 13687-13692.

15. Cassera MB, Gozzo FC, D'Alexandri FL, Merino EF, del Portillo HA, Peres VJ, et al. The methylerythritol phosphate pathway is functionally active in all intraerythrocytic stages of Plasmodium falciparum. J Biol Chem 2004; 279: 5174951759.

16. Wrenger $\mathrm{C}$, Eschbach $\mathrm{ML}$, Muller IB, Warnecke $\mathrm{D}$, Walter $\mathrm{RD}$. Analysis of the vitamin B6 biosynthesis pathway in the human malaria parasite Plasmodium falciparum. J Biol Chem 2005; 280: 5242-5248.

17. Knockel J, Muller IB, Bergmann B, Walter RD, Wrenger C. The apicomplexan parasite Toxoplasma gondii generates pyridoxal phosphate de novo. Mol Biochem Parasitol 2007; 152: 108-111.

18. Gengenbacher M, Fitzpatrick TB, Raschle T, Flicker K, Sinning I, Muller S, et al. Vitamin B6 biosynthesis by the malaria parasite Plasmodium falciparum: biochemical and structural insights. J Biol Chem 2006; 281: 3633-3641.

19. Bauer JA, Bennett EM, Begley TP, Ealick SE. Three-dimensional structure of YaaE from Bacillus subtilis, a glutaminase implicated in pyridoxal-5'-phosphate biosynthesis. $J$ Biol Chem 2004; 279: 2704-2711.

20. Zhu J, Burgner JW, Harms E, Belitsky BR, Smith JL. A new arrangement of (beta/alpha) 8 barrels in the synthase subunit of PLP synthase. J Biol Chem 2005; 280: 27914-27923.

21. Zein F, Zhang Y, Kang YN, Burns K, Begley TP, Ealick SE. Structural insights into the mechanism of the PLP synthase holoenzyme from Thermotoga maritima. Biochemistry 2006; 45: 14609-14620.

22. Strohmeier M, Raschle T, Mazurkiewicz J, Rippe K, Sinning I, Fitzpatrick TB, et al. Structure of a bacterial pyridoxal 5'phosphate synthase complex. Proc Natl Acad Sci U S A 2006; 103: 19284-19289.

23. Raschle T, Arigoni $\mathrm{D}$, Brunisholz R, Rechsteiner $\mathrm{H}$, Amrhein N, Fitzpatrick TB. Reaction mechanism of pyridoxal 5'-phosphate synthase. Detection of an enzyme-bound chromophoric intermediate. J Biol Chem 2007; 282: 6098-6105.

24. Kerry JA, Rohde M, Kwok F. Brain pyridoxal kinase. Purification and characterization. Eur J Biochem 1986; 158: 581585.

25. Yang $Y$, Zhao G, Winkler ME. Identification of the pdxK gene that encodes pyridoxine (vitamin B6) kinase in Escherichia coli K-12. FEMS Microbiol Lett 1996; 141: 89-95.

26. Scott TC, Phillips MA. Characterization of Trypanosoma brucei pyridoxal kinase: purification, gene isolation and expression in Escherichia coli. Mol Biochem Parasitol 1997; 88: 1-11.

27. Park JH, Burns K, Kinsland C, Begley TP. Characterization of two kinases involved in thiamine pyrophosphate and pyridoxal phosphate biosynthesis in Bacillus subtilis: 4amino-5-hydroxymethyl-2methylpyrimidine kinase and pyridoxal kinase. J Bacteriol 2004; 186: 1571-1573.

28. Friedrich W. Vitamins. Berlin: Walter de Gruyter; 1988.

29. Pohl M, Sprenger GA, Muller M. A new perspective on thiamine catalysis. Curr Opin Biotechnol 2004; 15: 335-342. 30. Mizote T, Tsuda M, Smith DD, Nakayama H, Nakazawa T.
Cloning and characterization of the thiD/J gene of Escherichia coli encoding a thiamin-synthesizing bifunctional enzyme, hydroxymethylpyrimidine kinase/phosphomethylpyrimidine kinase. Microbiology 1999; 145 (Part 2): 495-501.

31. Reddick JJ, Kinsland C, Nicewonger R, Christian T, Downs $\mathrm{DM}$, Winkler ME, et al. Overexpression, purification and characterization of two pyrimidine kinases involved in the biosynthesis of thiamine: 4-amino-5-hydroxymethyl-2-methylpyrimidine kinase and 4-amino-5-hydroxymethyl-2-methylpyrimidine phosphate kinase. Tetrahedron 1998; 54: 15983-15991.

32. Begley TP, Downs DM, Ealick SE, McLafferty FW, Van Loon AP, Taylor S, et al. Thiamin biosynthesis in prokaryotes. Arch Microbiol 1999; 171: 293-300.

33. Wrenger C, Eschbach ML, Muller IB, Laun NP, Begley TP, Walter RD. Vitamin B1 de novo synthesis in the human malaria parasite Plasmodium falciparum depends on external provision of 4-amino-5-hydroxymethyl-2-methylpyrimidine. Biol Chem 2006; 387: 41-51.

34. Zhang Y, Taylor SV, Chiu HJ, Begley TP. Characterization of the Bacillus subtilis thiC operon involved in thiamine biosynthesis. J Bacteriol 1997; 179: 3030-3035.

35. Hohmann S, Meacock PA. Thiamin metabolism and thiamin diphosphate-dependent enzymes in the yeast Saccharomyces cerevisiae: genetic regulation. Biochim Biophys Acta 1998; 1385: 201-219.

36. Wightman R, Meacock PA. The THI5 gene family of Saccharomyces cerevisiae: distribution of homologues among the hemiascomycetes and functional redundancy in the aerobic biosynthesis of thiamin from pyridoxine. Microbiology 2003; 149: 1447-1460.

37. Morett E, Korbel JO, Rajan E, Saab-Rincon G, Olvera L, Olvera $M$, et al. Systematic discovery of analogous enzymes in thiamin biosynthesis. Nat Biotechnol 2003; 21: 790-795.

38. Mizote $\mathrm{T}$, Nakayama $\mathrm{H}$. The thiM locus and its relation to phosphorylation of hydroxyethylthiazole in Escherichia coli. J Bacteriol 1989; 171: 3228-3232.

39. Settembre EC, Dorrestein PC, Zhai H, Chatterjee A, McLafferty FW, Begley TP, et al. Thiamin biosynthesis in Bacillus subtilis: structure of the thiazole synthase/sulfur carrier protein complex. Biochemistry 2004; 43: 11647-11657.

40. Peapus DH, Chiu HJ, Campobasso N, Reddick JJ, Begley TP, Ealick SE. Structural characterization of the enzymesubstrate, enzyme-intermediate, and enzyme-product complexes of thiamin phosphate synthase. Biochemistry 2001; 40: 10103-10114.

41. Nosaka $\mathrm{K}$, Kaneko $\mathrm{Y}$, Nishimura H, Iwashima A. Isolation and characterization of a thiamin pyrophosphokinase gene, THI80, from Saccharomyces cerevisiae. J Biol Chem 1993; 268: 17440-17447.

42. Baker LJ, Dorocke JA, Harris RA, Timm DE. The crystal structure of yeast thiamin pyrophosphokinase. Structure 2001; 9: 539-546.

43. Bozdech Z, Ginsburg H. Data mining of the transcriptome of Plasmodium falciparum: the pentose phosphate pathway and ancillary processes. Malar J 2005; 4: 17.

44. Ralph SA, van Dooren GG, Waller RF, Crawford MJ, Fraunholz MJ, Foth BJ, et al. Tropical infectious diseases: metabolic maps and functions of the Plasmodium falciparum apicoplast. Nat Rev Microbiol 2004; 2: 203-216. 
45. Eschbach ML, Muller IB, Gilberger TW, Walter RD, Wrenger C. The human malaria parasite Plasmodium falciparum expresses an atypical $\mathrm{N}$-terminally extended pyrophosphokinase with specificity for thiamine. Biol Chem 2006; 387: 1583-1591.

46. Muller S, Kappes B. Vitamin and cofactor biosynthesis pathways in Plasmodium and other apicomplexan parasites. Trends Parasitol 2007; 23: 112-121.

47. Foth BJ, Stimmler LM, Handman E, Crabb BS, Hodder AN, McFadden Gl. The malaria parasite Plasmodium falciparum has only one pyruvate dehydrogenase complex, which is located in the apicoplast. Mol Microbiol 2005; 55: 39-53.

48. McMillan PJ, Stimmler LM, Foth BJ, McFadden GI, Muller $\mathrm{S}$. The human malaria parasite Plasmodium falciparum possesses two distinct dihydrolipoamide dehydrogenases. $\mathrm{Mol}$ Microbiol 2005; 55: 27-38.

49. Marobbio CM, Vozza A, Harding M, Bisaccia F, Palmieri F, Walker JE. Identification and reconstitution of the yeast mitochondrial transporter for thiamine pyrophosphate. EMBO J 2002; 21: 5653-5661.

50. Zilles JL, Croal LR, Downs DM. Action of the thiamine antagonist bacimethrin on thiamine biosynthesis. J Bacteriol 2000; 182: 5606-5610.

51. Lawhorn BG, Gerdes SY, Begley TP. A genetic screen for the identification of thiamin metabolic genes. $\mathrm{J}$ Biol Chem 2004; 279: 43555-43559.
52. Divo AA, Geary TG, Davis NL, Jensen JB. Nutritional requirements of Plasmodium falciparum in culture. I. Exogenously supplied dialyzable components necessary for continuous growth. J Protozool 1985; 32: 59-64.

53. Tazuya K, Adachi Y, Masuda K, Yamada K, Kumaoka H. Origin of the nitrogen atom of pyridoxine in Saccharomyces cerevisiae. Biochim Biophys Acta 1995; 1244: 113-116.

54. Zeidler J, Ullah N, Gupta RN, Pauloski RM, Sayer BG, Spenser ID. 2'-Hydroxypyridoxol, a biosynthetic precursor of vitamins $\mathrm{B}(6)$ and $\mathrm{B}(1)$ in yeast. J Am Chem Soc 2002; 124: $4542-4543$.

55. Zeidler J, Sayer BG, Spenser ID. Biosynthesis of vitamin B1 in yeast. Derivation of the pyrimidine unit from pyridoxine and histidine. Intermediacy of urocanic acid. J Am Chem Soc 2003; 125: 13094-13105.

56. Sherman IW. Biochemistry of Plasmodium (malarial parasites). Microbiol Rev 1979; 43: 453-495.

57. Gardner MJ, Hall N, Fung E, White O, Berriman M, Hyman RW, et al. Genome sequence of the human malaria parasite Plasmodium falciparum. Nature 2002; 419: 498-511.

58. Schopfer WH. Plants and vitamins. Waltham: Chronica Botanica Company; 1949.

59. Rodionov DA, Vitreschak AG, Mironov AA, Gelfand MS. Comparative genomics of thiamin biosynthesis in procaryotes. New genes and regulatory mechanisms. J Biol Chem 2002; 277: 48949-48959. 